\title{
Association between advanced glycation end-products and functional performance in Alzheimer's disease and mixed dementia
}

\author{
Hans Drenth, ${ }^{1,2}$ Sytse U. Zuidema, ${ }^{3}$ Wim P. Krijnen, ${ }^{1}$ Ivan Bautmans, ${ }^{4}$ Cees van der \\ Schans ${ }^{1,5,6}$ and Hans Hobbelen ${ }^{1,3}$ \\ ${ }^{1}$ Research Group Healthy Ageing, Allied Healthcare and Nursing, Hanze University of Applied Sciences, Groningen, the Netherlands \\ ${ }^{2}$ Zuid Oost Zorg, Organisation for Elderly Care, Drachten, the Netherlands \\ ${ }^{3}$ Department of General Practice and Elderly Care Medicine, University of Groningen, University Medical Center Groningen, Groningen, the Netherlands \\ ${ }^{4}$ Frailty in Ageing Research Group and Gerontology Department, Vrije Universiteit Brussel, Brussels, Belgium \\ ${ }^{5}$ Department of Rehabilitation Medicine, University of Groningen, University Medical Center Groningen, Groningen, the Netherlands \\ ${ }^{6}$ Health Psychology Research, University of Groningen, University Medical Center Groningen, Groningen, the Netherlands
}

Background: People with Alzheimer's disease (AD) experience, in addition to the progressive loss of cognitive functions, a decline in functional performance such as mobility impairment and disability in activities of daily living (ADL). Functional decline in dementia is mainly linked to the progressive brain pathology. Peripheral biomechanical changes by advanced glycation end-products (AGEs) have been suggested but have yet to be thoroughly studied.

Methods: A multi-center, longitudinal, one-year follow-up cohort study was conducted in 144 people with early stage AD or mixed Alzheimer's/Vascular dementia. Linear mixed model analyses was used to study associations between AGE-levels (AGE reader) and mobility (Timed Up and Go), and ADL (Groningen Activity Restriction Scale and Barthel index), respectively.

Results: A significant association between AGE levels and mobility ( $\beta=3.57,95 \% \mathrm{CI}$ : $1.43-5.73)$ was revealed; however, no significant association between AGE levels and ADL was found. Over a one-year time span, mean AGE levels significantly increased, and mobility and ADL performance decreased. Change in AGE levels was not significantly correlated with change in mobility.

Conclusions: This study indicates that high AGE levels could be a contributing factor to impaired mobility but lacks evidence for an association with ADL decline in people with early stage $\mathrm{AD}$ or mixed dementia. Future research is necessary on the reduction of functional decline in dementia regarding the effectiveness of interventions such as physical activity programs and dietary advice possibly in combination with pharmacologic strategies targeting AGE accumulation.

Key words: Alzheimer's disease, dementia, functional mobility, activities of daily living, biomarker, advanced glycation end-products

\section{Background}

People with Alzheimer's disease (AD) experience, in addition to the progressive loss of cognitive functions, a decline in functional performance such as mobility impairment and disability in activities of daily living (ADL) (American Psychiatric Association, 1994). In early stage $\mathrm{AD}$, there are already decreases in step length and walking velocity and,

\footnotetext{
Correspondence should be addressed to: Hans Drenth, Research Group Healthy Ageing, Allied Healthcare and Nursing, Hanze University of Applied Sciences, PO Box 3109, 9701 DC, Groningen, the Netherlands. Email: j.c.drenth@pl.hanze.nl. Received 15 Feb 2017; revision requested 21 Mar 2017; revised version received 22 Apr 2017; accepted 27 Apr 2017. First published online 25 May 2017.
}

in early vascular dementia (VaD), a small step gait, slow stepping, ataxic gait, and unsteadiness is found (Scherder et al., 2011). Impairment in basic ADL (BADL) functions (i.e., bathing, toileting, feeding, and dressing) in early dementia is more reliant on decline in motor function, whereas impairment in instrumental ADL (IADL) functions (i.e. housekeeping, cooking and finance management) is more reliant on cognition decline (Martyr and Clare, 2012). Furthermore, it is known that specifically in the early stages initiating certain IADL (i.e. preparing a meal, finance management) is found to be more strongly impaired than their performance (Giebel et al., 2017). Decline in functional performance contributes to an increase 
in care burden and a decrease in the quality of life (Andersen et al., 2004). Lower level and accelerated decline of functional performance is suggested to predict the subsequent development of mild cognitive impairment and $\mathrm{AD}$ and can precede cognitive impairment by several years (Buchman and Bennett, 2011). Ramakers et al. determined that, even five years prior to the dementia diagnosis, walking impairments were significantly higher in pre-clinical people with dementia compared to the control group (Ramakers et al., 2007). A decline in functional performance could be predicted with biomarkers; one of the proposed biomarkers is advanced glycation end-products (AGEs). AGE accumulation contributes to the age-related decline of the functioning of cells and tissues in normal aging (Rahmadi et al., 2011). Interestingly, AD is related to higher concentrations of AGEs (Rahmadi et al., 2011; Li et al., 2012). AGEs, therefore, are a potential biomarker and an accompanying risk factor for the decline of functional performance in people with $\mathrm{AD}$ and mixed dementia $(\mathrm{AD} / \mathrm{VaD})$.

\section{Advanced glycation end products}

Decline in functional performance in dementia is primarily associated with central mechanisms as a result of progressive brain pathology. Peripheral biomechanical changes have been suggested but have yet to be thoroughly studied. Studies in stroke patients show that immobility is associated with adaptive mechanical and morphological changes in muscle tissue in which muscles become stiffer (Farmer and James, 2001). A review comprising eight studies suggests that, in participants without dementia, AGEs-induced muscle biomechanical changes contribute to a decline in walking abilities and in BADL as well as physical frailty (Drenth et al., 2016). It was recently found that AGEs are associated with the presence and severity of paratonia, a distinctive form of hypertonia/movement stiffness in dementia, which suggests that peripheral biomechanical changes contribute to movement stiffness in early stage dementia (Drenth et al., 2017).

AGEs are formed by the non-enzymatic condensation of a reducing sugar with proteins or lipids and accumulate in hyperglycaemic environments. The accumulation of AGEs is an element of normal metabolism that accelerates in a wide variety of diseases and during normal aging (Ahmed and Thornalley, 2007; Rahmadi et al., 2011). AGEs can be categorized into fluorescent cross-linking, non-fluorescent cross-linking, fluorescent non-crosslinking, and non-fluorescent noncrosslinking (Meerwaldt et al., 2004). AGEs tissue accumulation can be estimated non-invasively by skin auto-fluorescence (SAF) with an AGE reader by utilizing the fluorescent properties of specific AGEs that correlate with non-fluorescent AGEs (Meerwaldt et al., 2004).

The cross-linking of long-lived proteins, particularly collagen, is responsible for increasing mechanical stiffness and loss of elasticity (Avery and Bailey, 2005). Non cross-linking effects occur by the binding of AGEs to the receptor for AGEs (RAGE) that incites the production of proinflammatory cytokines and free radicals. At the central level, interaction between AGEs, Amyloidbeta, and tau-protein have been ascertained to affect neuronal function (Ahmed and Thornalley, 2007). At the peripheral level, this AGE/RAGE interaction affects collagen tissue and may play a role in sarcopenia (loss of muscle mass and strength) through upregulated inflammation and endothelial dysfunction in the intra-muscular microcirculation (Payne, 2006).

These peripheral and central effects of AGEs may have a direct or indirect influence on muscle function and functional performance decline; however, this has not been studied in people with dementia. Early detection could initiate interventions targeting AGE accumulation, such as physical activity programs and dietary advice possibly in combination with pharmacologic strategies (Magelhaes et al., 2008; Puyvelde et al., 2014; Nenna et al., 2015) to attenuate functional decline, affording people with dementia longer independence. The aim, therefore, was to investigate the association between AGEs and functional performance in people with $\mathrm{AD}$ and mixed dementia $(\mathrm{AD} / \mathrm{VaD})$.

\section{Method}

\section{Design}

The study was designed as a multi-center, longitudinal, one-year observational follow-up cohort study with three assessments: at baseline, after six months, and after 12 months.

\section{Study population}

Participants, selected from 24 dementia day-care centers in the Netherlands, were considered to be eligible for inclusion when they satisfied four criteria: (1) an established diagnosis of Alzheimer's disease (AD) or mixed Alzheimer's/Vascular dementia ( $\mathrm{AD} / \mathrm{VaD}$ ) according to DSM-IV criteria (American Psychiatric Association, 1994); (2) a score of stage five or lower on the Global Deterioration Scale (GDS) (Reisberg et al., 1982); (3) able to walk $10 \mathrm{~m}$ (a walking aid was allowed); 
and (4) having a light-colored (Caucasian) skin due to the limitations of the AGE-reader device (Meerwaldt et al., 2004). Written informed consent was obtained from the participants or their legal representatives. The medical ethical committee of the University Medical Centre Groningen approved the study (NL43641.042.13).

Design and study population are derived from the PARAGE study (Drenth et al., 2017) and described more extensively.

\section{Outcome measures}

Mobility

The participant's functional mobility was assessed with the Timed Up and Go (TUG). It measures the amount of time a participant takes to stand up from a chair (with an approximate height of $46 \mathrm{~cm}$ ) and walk $3 \mathrm{~m}$, and then turn around a cone, walk back to the chair, and sit down. The TUG is a validated and reliable test for people with dementia. A score of $20 \mathrm{sec}$ or more indicates the presence or increase of additional mobility problems (Ries et al., 2009). TUG measurements were obtained by experienced physiotherapists followed by the AGEs measurements assessed by the main researcher at each visit. Prior to the study, the physical therapists were trained by the main researcher on how to perform the measurements.

ADL

To assess the broad construct of ADL, the outcome from the participants' perspectives is measured with the Groningen Activity Restriction Scale (GARS) (Kempen et al., 1993) and from the daycare center staffs' perspective with the Barthel index (BI) (Collin et al., 1988). The GARS has been ascertained as being valid for measuring disabilities in personal care. With the GARS, the participants are questioned about their capabilities in personal care on two subscales. The first subscale is regarding BADL (11 items), and the second subscale relates to IADL ( 7 items). The answers are rated on a four-point scale with 1 meaning no support and 4 meaning only with help. A lower score on the combined subscales indicates more ADL independence with 18 as the minimum score (Metzelthin et al., 2010). The BI is a valid and reliable measurement for assessing a person's ability of self-care. Ten items regarding BADL and mobility are rated by the participant's caregiver based on the amount of assistance required to complete each activity. A higher score indicates more BADL independence with 20 as the maximum score (Collin et al., 1988). The questionnaires (GARS and BI) were administered by key staff personnel from the day- care centers within the same week that the AGEs measurements were taken. Before the study, the participating day-care staff was trained by the primary researcher to perform the measurements.

\section{AGE LEVEL}

AGE levels are measured with the AGE reader (Diagnoptics, Groningen, the Netherlands) that is a desktop device measuring fluorescent skin AGEs and is reported to be valid and reliable for the quantification of AGEs tissue accumulation (Meerwaldt et al., 2004). The skin of the forearm is illuminated by a light source in the AGE reader through a $1-\mathrm{cm}^{2}$ hole that is guarded against surrounding light. Excitation light in the wavelength range of 300-420 $\mathrm{nm}$ (peak excitation $\sim 350 \mathrm{~nm}$ ) is projected onto the skin surface. The intensity of light emitted from the skin in the wavelength range of $420-600 \mathrm{~nm}$ is measured with a spectrometer. SAF is calculated as the ratio between the emission light and the excitation light using the AGE reader software and expressed in arbitrary units (AU). A high SAF score corresponds to a high tissue AGEs level (Meerwaldt et al., 2004). The measurements were performed on the forearm without sweat, skin lotions, or visible skin abnormalities and with the assessor being blinded for functional performances scores. All AGE reader measurements were performed at room temperature in a standardized semi-dark environment with the participants in a seated position and the volar side of the right forearm placed on top of the AGE reader. The mean of three consecutive measurements was used for analysis.

\section{OTHER VARIABLES}

Dementia characteristics were provided by the general practitioner (GP) or the local physician. Cognitive functioning was measured by experienced psychologists or physicians with the MiniMental State Examination (MMSE). The MMSE is an 11-item questionnaire with a maximum score of 30 , which indicates no cognitive decline, and a minimum score of 0 , which indicates a very severe cognitive decline (Folstein et al., 1975). Dementia severity was categorized by key staff personnel from the day-care centers using the GDS that identifies seven clinically recognizable stages from normal (no dementia) to severe dementia (Reisberg et al., 1982). Paratonia was diagnosed by physical therapists with the Paratonia Assessment Instrument (PAI) at each visit. The PAI is a reliable and valid dichotomous assessment instrument with which an examiner can establish the presence (or absence) of paratonia by successively moving all four limbs passively in flexion and extension, while 
the participant is in a sitting position (Hobbelen et al., 2008).

The use of medication and the presence of comorbidities (ICD-9 classification) were retrieved from the participant's medical records and GP files. The use of five or more medications was labeled as polypharmacy (World Health Organisation, 2014).

\section{Statistical analyses}

SAMPle Size Calculation

A mixed model sample size calculation (Liu and Liang, 1997) was based upon a 0.5 correlation between repeated measurements, 0.3 variance of the random intercept, 0.3 residual variance, a true effect size of 0.5 , a desired power of $80 \%$, and a two-sided alpha of 0.05 . This resulted in a total sample size of 152 . Addressing an eventual $10 \%$ withdrawal resulted in a required total number of approximately 165 participants. Baseline characteristics are depicted by descriptive statistics and presented in Table 1 .

\section{Association between AGE Levels and}

MOBILITY AND ADL

To investigate the association between AGE levels on mobility and ADL(BADL/IADL), linear mixed model analyses (LMM) was employed, estimated by restricted maximum likelihood taking the TUG, GARS, and BI measurements at each of the three visits as the response variable.

The models controlled statistically for the fixed effects of AGEs level, time (visit), gender, age, polypharmacy, dementia duration, cognition (MMSE), paratonia, chronic kidney disease (CKD), cardiovascular disease (CVD), cerebral vascular disease (CeVD), and diabetes mellitus (DM) (Rahmadi et al., 2011). Participants were taken as random effects. Backward model selection was utilized to identify statistically significant explanatory variables. During this process, AGEs level and time were always retained in order to study the size of their effect.

To investigate change in mean over one year (between the last visit and baseline) on AGE levels and TUG, GARS, and BI, paired sample $t$ tests were performed. Pearson's $R$ was calculated to investigate the association between change in AGE levels and change in the previously described variables. To further explore specific longitudinal effects, a linear regression was used where the change of the described scores between visit three and one was used as a response variable and changes of AGEs level and baseline characteristics (described above) as explanatory variables.
Data were analyzed using $\mathrm{R}$ version 3.2 .0 and SPSS version 22, taking a p-value $<0.05$ as statistically significant.

\section{Results}

From the 244 people with dementia approached to take part in the study, 87 were not included due to not satisfying the inclusion criteria; 13 participants withdrew informed consent prior to the baseline assessment. Finally, 144 participants were included at baseline. After one year, 26 participants (18\%) were lost to follow-up: 11 deceased, while 15 were transferred to unknown addresses or became too ill to be reassessed (Figure 1). For comorbidity and medication use in $11 \%$ of the participants $(n=$ 16), no information was accessible in the medical records (information derived from the PARAGE study (Drenth et al., 2017)).

The baseline characteristics are summarized in Table 1.

\section{Association between AGEs and mobility}

Table 2 indicates that, after correction for age, polypharmacy, CKD, CVD, and MMSE, functional mobility (TUG) was significantly associated with the AGE levels $(\beta=3.57, \mathrm{p}=0.001,95 \% \mathrm{CI}: 1.43-$ 5.73) and with the progression of dementia over a one-year time span $(\beta=3.73, \mathrm{p}=0.001,95 \% \mathrm{CI}$ : 1.46-5.91)

\section{Association between AGEs and ADL}

Table 3 indicates that, after removing covariates not being statistically significant, the AGE levels did not have a significant effect on ADL; however, the GARS (BADL/IADL) and BI were associated with the progression of dementia over one-year time span $(\beta=2.58, \mathrm{p}<0.001,95 \% \mathrm{CI}: 1.47-3.67$, $\beta=1.98, \mathrm{p}<0.001,95 \% \mathrm{CI}: 0.79-3.17$ and $\beta$ $=-2.00, \mathrm{p}<0.001,95 \% \mathrm{CI}:-2.58$ to -1.42 , respectively).

The one-year development of AGE levels and functional mobility and ADL (BADL/IADL) is presented in Table 4. From the 118 participants $(82 \%)$ who completed this study, the longitudinal data over one year indicates that there was a significant increase in the overall AGE levels as well as TUG and GARS scores and a significant decrease in BI scores. Changes in functional performance (TUG, GARS, and BI) between baseline and visit three indicated no significant correlation with changes in the AGE level over one year. The linear regression models show that change in AGEs level is not predictive for changes 
Table 1. Baseline characteristics

\begin{tabular}{|c|c|c|}
\hline & \multicolumn{2}{|c|}{ TOTAL $N=144$} \\
\hline & FREQUENCY & RANGE MIN-MAX \\
\hline Male, $n(\%)$ & $63(43.7 \%)$ & \\
\hline Age, years & $80.7(7.7)$ & $53-98$ \\
\hline Dementia duration, months ${ }^{a}$ & $29.8(35.9)$ & $1-252$ \\
\hline Co-morbidities, $n^{\mathrm{a}}$ & $1.18(1.1)$ & $0-4$ \\
\hline $\mathrm{AD}, n(\%)^{\mathrm{a}}$ & $107(83.6 \%)$ & \\
\hline Mixed $\mathrm{AD} / \mathrm{VaD}, n(\%)^{\mathrm{a}}$ & $21(16.4 \%)$ & \\
\hline $\mathrm{AD}$ or Mixed $\mathrm{AD} / \mathrm{VaD}, n(\%)^{\mathrm{b}}$ & $16(11.11 \%)$ & \\
\hline CVD, $n(\%)^{\mathrm{a}}$ & $41(32.0 \%)$ & \\
\hline CeVD (CVA, TIA), $n(\%)^{\mathrm{a}}$ & $22(17.2 \%)$ & \\
\hline $\mathrm{DM}, n(\%)^{\mathrm{a}}$ & $37(28.9 \%)$ & \\
\hline Cancer, $n(\%)^{\mathrm{a}}$ & $14(11.0 \%)$ & \\
\hline COPD, $n(\%)^{\mathrm{a}}$ & $14(11.0 \%)$ & \\
\hline $\mathrm{CKD}, n(\%)^{\mathrm{a}}$ & $11(8.6 \%)$ & \\
\hline Systemic, $n(\%)^{\mathrm{a}}$ & $8(6.3 \%)$ & \\
\hline Digestive tract, $n(\%)^{\mathrm{a}}$ & $6(4.7 \%)$ & \\
\hline Polypharmacy ( $\geq 5$ meds), $n(\%)^{\mathrm{a}}$ & $71(55.5 \%)$ & \\
\hline MMSE, score $0-30$ & $19.4(5.4)$ & $6-29$ \\
\hline GDS, score $1-7$ & $3.84(1.0)$ & $2-5$ \\
\hline Paratonia, PAI Yes & $60(41.7 \%)$ & \\
\hline TUG, seconds & $17(9.7)$ & $7-65$ \\
\hline GARS BADL, score $11-44$ & $16.0(5.7)$ & $1-37$ \\
\hline GARS IADL, score 7-28 & $16.8(6.3)$ & $7-28$ \\
\hline GARS total, score $18-72$ & $32.9(10.7)$ & $18-65$ \\
\hline BI, score $0-20$ & $16(3.5)$ & $6-20$ \\
\hline AGE levels, SAF (AU) & $2.8(0.7)$ & $0.4-4.9$ \\
\hline
\end{tabular}

Frequency data represent mean values (SD) unless indicated otherwise.

${ }^{a}$ Based on GP medical files $n=128$, b based on chart diagnoses provided by day-care staff personnel for study inclusion.

CVD: Cardio Vascular disease, CeVD: Cerebral Vascular disease, CVA: Cerebral Vascular Accident, TIA: Transient Ischemic Attack, DM: Diabetes Mellitus, COPD: Chronic Obstructive Pulmonary Disease, CKD: Chronic Kidney Disease, MMSE: Mini Mental State Exam, GDS: Global Deterioration Scale, TUG: Timed Up and Go, GARS: Groninger Activity Restriction Scale, BADL: Basic Activities Daily Living, IADL: Instrumental Activities Daily living BI: Barthel Index, AGE's: Advanced Glycation End-products, SAF: Skin AutoFluoresce (AGE reader) AU: Arbitrary Units i.e., the output units of the AGE reader.

in functional performance, not even after adjusting for baseline characteristics.

\section{Discussion}

Over a one-year time span, the AGE levels significantly increased and mobility, BADL and IADL performance decreased. This study shows that AGE levels are significantly associated with functional mobility, however, not with BADL or IADL in people experiencing early stage $A D$ and mixed Alzheimer's/Vascular disease ( $\mathrm{AD} / \mathrm{VaD})$.

Although change in AGE levels was not significantly related to change in functional mobility, mixed model analyses revealed a significant combined time and between participant effects of AGEs on functional mobility. Participants with higher AGE levels scored higher on the TUG, indicating lower functional mobility. The encountered Beta effect means that, with every unit of AGE reader increase, the time to perform the TUG increases with $3.57 \mathrm{sec}$. The TUG measures functionality when transferring from sitting to standing, turning, and walking speed. A TUG score greater than $20 \mathrm{sec}$ indicates mobility problems and slow walking speed that is associated with a wide range of adverse health consequences such as frailty, falls, disability, hospitalization, and institutionalization (Ries et al., 2009). It is suggested that every decrease of $0.1 \mathrm{~m} / \mathrm{sec}$ in walking speed already increases the risk of these negative health outcomes (Graham et al., 2008). With a typical clinical $\mathrm{AD}$ duration of eight to ten years (Bird, 1993), extrapolating the Beta effect of $3.57 \mathrm{sec}$ would become even more relevant. 
Table 2. Association between AGEs and mobility (TUG) corrected for possible explanatory variables obtained after backward model selection

\begin{tabular}{|c|c|c|c|c|}
\hline & \multicolumn{4}{|c|}{ MовILITY (TUG) } \\
\hline & \multirow[b]{2}{*}{ ESTIMATE } & \multirow[b]{2}{*}{ P VALUE } & \multicolumn{2}{|c|}{$95 \% \mathrm{CI}$} \\
\hline & & & LOWER LIMIT & UPPER LIMIT \\
\hline (Intercept) & -14.33 & 0.196 & -35.59 & 6.90 \\
\hline Age & 0.42 & 0.002 & 0.16 & 0.68 \\
\hline Polypharmacy & 4.75 & 0.020 & 0.88 & 8.60 \\
\hline CKD & -8.75 & 0.012 & -15.31 & -2.19 \\
\hline MMSE & -0.76 & $<0.001$ & -1.11 & -0.40 \\
\hline AGE levels & 3.57 & 0.001 & 1.43 & 5.73 \\
\hline Visit2* & 2.41 & 0.026 & 0.30 & 4.50 \\
\hline Visit3* & 3.73 & 0.001 & 1.46 & 5.91 \\
\hline
\end{tabular}

Response variable: Timed Up and Go (TUG) in seconds.

Explanatory variable: AGE levels (SAF) AU.

CKD: Chronic Kidney Disease, MMSE: Mini-Mental State Exam, AGE: Advanced Glycation End-product.

${ }^{*}$ Visit effects with respect to baseline (Visit1).

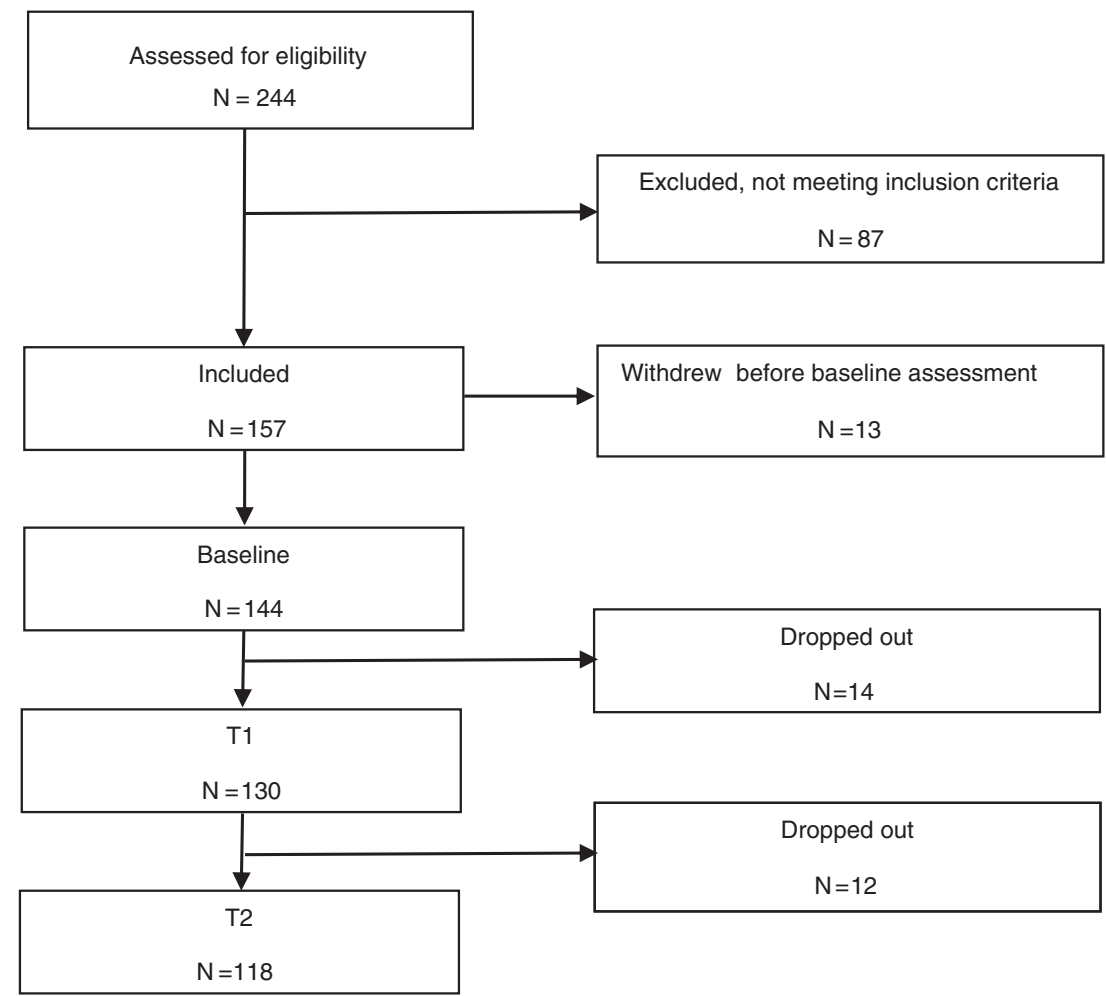

Figure 1. Study flow chart.

The result from this study is consistent with studies describing the effect of AGEs on the decline of walking abilities and contributes to the increasing evidence that decline in functional mobility can be attributed to the effects of AGEs on muscle tissue (Drenth et al., 2016). The loss of skeletal muscle mass and weakness is an important contributor to functional decline. Both muscle weakness and walking impairment are prominent characteristics of physical frailty (Sternberg et al., 2011), suggesting that high AGEs level are a contributing factor to physical frailty. It remains ambiguous whether the association between high AGE levels and functional mobility decline exists because AGEs damage muscle tissue or whether loss of physical activity due to functional mobility decline influences AGEs accumulation. Future research is necessary to study this more in depth. 


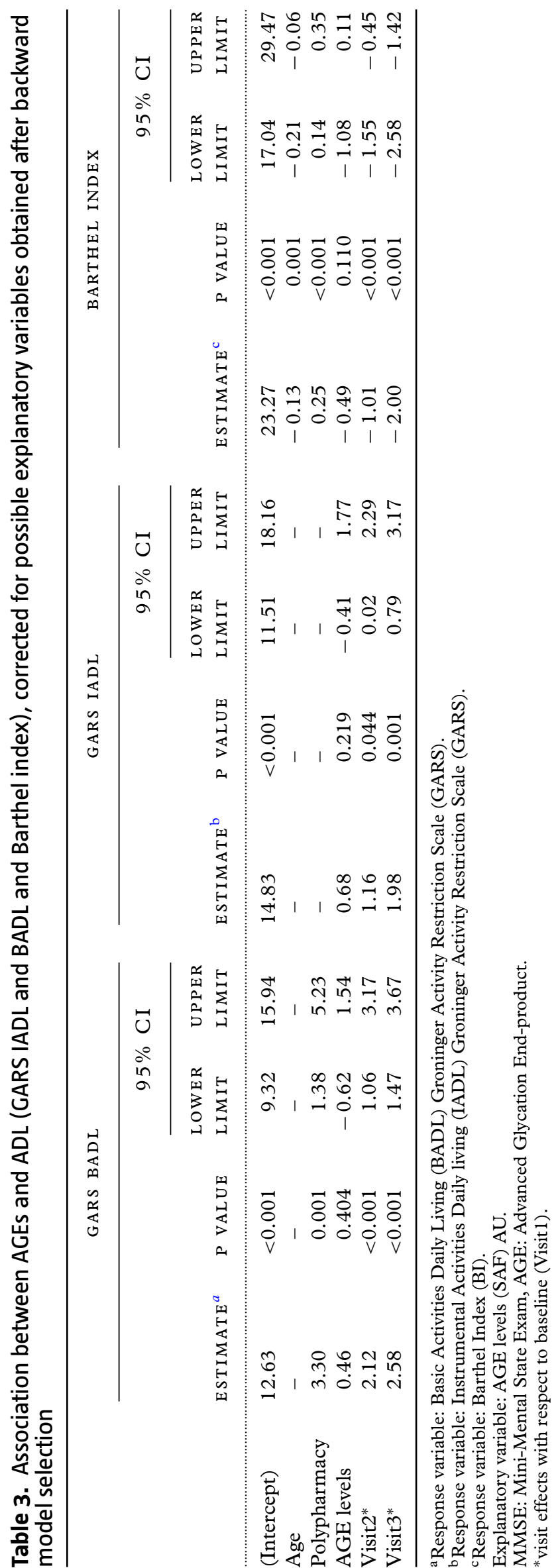

In accordance with the results of this study, it would be interesting to investigate whether decline in functional mobility can be attenuated by reducing AGEs levels. Excessive elevation of glucose concentration, such as in DM, most likely accelerates the glycation of proteins (Ahmed and Thornalley, 2007; Rahmadi et al., 2011). Intensive glycaemic control may be a method to decrease AGEs formation. AGEs are not only produced endogenously, but are also spontaneously generated in standard diets (Magelhaes et al., 2008). Therefore, dietary intake is a possible factor that can be influenced. In order to lower daily AGEs intake, it is suggested that foods rich in sugar and fat as well as those prepared by frying or grilling should be avoided (Puyvelde et al., 2014). However, evidence of the harmful effects of long-term exposure to dietary AGEs is currently inconclusive (Puyvelde et al., 2014). Additionally, regular physical activity has demonstrated a correlation with reduced glycation and AGE formation; however, the optimal exercise modalities still remain unclear (Magelhaes et al., 2008). In addition, pharmacologic strategies to prevent AGE formation or AGE accumulation are being studied, but results show conflicting evidence and additional research is necessary (Nenna et al., 2015).

An association between AGE levels and decline in $\mathrm{ADL}$ (BADL/IADL) was not determined. This is in contrast with a large cohort $(n=3,373)$ study by Whitson et al. that reported an association between serum AGE levels and the time to incident BADL disability in heathy participants over 14 years $(\mathrm{HR}=1.10,95 \% \mathrm{CI}: 1.05-1.15)$ (Whitson et al., 2014). Although it is likely that impaired muscle function - through AGEs-induced muscle damage - can contribute to impaired BADL, the results from the current study did not confirm this in people with early stage dementia. Our sample size was possibly too small and a study duration of one year too brief to detect a decline in ADL, and/or the participants were less ADL independent at baseline. Further research with a longer follow up time is necessary to investigate if AGE levels are related to the deterioration of $\mathrm{ADL}$ during the course of $\mathrm{AD}$, mixed dementia $(\mathrm{AD} / \mathrm{VaD})$, or other forms of dementia.

The strengths of this study are its longitudinal design with three assessments and that participating personnel were well trained in using the measurements. A study sample representative for this population was also created by including participants from rural and urban areas who were dispersed across the Netherlands. This study also has a number of limitations. First, the initial 
Table 4. One-year development of AGE levels and mobility and ADL

\begin{tabular}{|c|c|c|c|c|c|c|c|c|c|}
\hline & \multicolumn{2}{|c|}{ VISIT 1} & \multicolumn{2}{|c|}{ VISIT 3} & \multirow{2}{*}{$\begin{array}{l}\text { P VALUE VISIT } \\
\text { 3-VISIT } 1\end{array}$} & \multirow[b]{2}{*}{$R$} & \multirow[b]{2}{*}{ P VALUE } & \multirow[b]{2}{*}{ BETA } & \multirow[b]{2}{*}{ P VALUE } \\
\hline & FREQUENCY & RANGE & FREQUENCY & RANGE & & & & & \\
\hline AGE levels & $2.8(0.7)$ & $0.4-4.9$ & $3.0(0.7)$ & $1.6-5.3$ & $<0.001$ & - & - & - & - \\
\hline TUG & $17(9.7)$ & $7-65$ & $20(15)$ & 6-103 & $<0.001$ & 0.002 & 0.984 & -1.44 & 0.487 \\
\hline GARS BADL & $16.1(5.7)$ & $1-37$ & $18.3(6.9)$ & $11-39$ & $<0.001$ & 0.053 & 0.569 & -0.81 & 0.428 \\
\hline GARS IADL & $16.8(6.3)$ & $7-28$ & $18.6(6.7)$ & $7-28$ & $<0.001$ & 0.053 & 0.570 & 0.18 & 0.896 \\
\hline Barthel index & $16(3.5)$ & $6-20$ & $14.2(4.5)$ & $1-20$ & $<0.001$ & 0.011 & 0.908 & 0.86 & 0.186 \\
\hline
\end{tabular}

Frequency data represent mean values (SD) unless indicated otherwise.

TUG: Timed Up and Go, GARS: Groninger Activity Restriction Scale, BADL: Basic Activities Daily Living, IADL: Instrumental Activities Daily living, and AGE: Advanced Glycation End-product.

$R$ : Pearson Correlation coefficient between change (delta visit 3 visit 1) in AGE levels and change (delta visit 3 visit 1 ) TUG, GARS, and BI scores.

Beta: Linear regression model between change (delta visit 3 visit 1) in AGE levels and change (delta visit 3 visit 1) TUG, GARS, and BI scores.

number of 152 participants from our a priori sample size calculation could not be included. However, the study still comprised a reasonable number of 144 participants for baseline and 118 for follow up analysis. Second, due to the limitation of the AGE reader, it was not possible to indicate what types of AGEs (e.g. crosslinking or non-crosslinking) are responsible for our findings. Future fundamental research is necessary to further explore this. Finally, the follow-up period of one year is possibly insufficient for detecting an association between changes in AGE levels and change in functional performance over time. Prolonging the study over several years and in a larger cohort could result in improved insight in the long-term effects of AGEs on functional performance. Further longitudinal studies over several years are needed to investigate a causal relationship.

In conclusion this study indicates that high AGE levels could be a contributing factor to the decline in functional mobility in addition to the progressive brain pathology, but lacks evidence for an association with ADL decline in people with early stage $\mathrm{AD}$ or mixed dementia. This result contributes to the increasing evidence that high AGE levels could affect functional mobility in the aging population. Future research is necessary into interventions such as physical activity programs and dietary advice targeting AGE accumulation possibly in combination with pharmacologic strategies to attenuate functional decline in those experiencing dementia.

\section{Conflict of interest}

None.

\section{Description of authors' roles}

H. Drenth is the first author and was responsible for the conception and design, the acquisition, analysis and interpretation of data, and drafting the manuscript. S. Zuidema has been involved in the conception and design and has been involved in reviewing the manuscript critically for important intellectual content. W. Krijnen was involved in the data analysis and reviewing the manuscript critically for important intellectual content. I. Bautmans has been involved in reviewing and revising the manuscript critically for important intellectual content. C. van der Schans has been involved in revising the manuscript critically for important intellectual content. H. Hobbelen has been involved in the conception and design, reviewing the manuscript critically for important intellectual content, and has given final approval of the version to be published. All authors read and approved the final manuscript.

\section{Acknowledgments}

This work was supported by the Dutch Alzheimer organisation (grant number WE.03-2012-09) and by regular funds of the Research Group Healthy Ageing, Allied Healthcare and Nursing, Hanze University Groningen, the Department of General Practice and Elderly Care Medicine, University Medical Center Groningen, the Frailty in Ageing Research Group and Gerontology Department, Vrije Universiteit Brussels and ZuidOostZorg, Organisation for Elderly Care, Drachten. The authors kindly acknowledge and thank the participants for their participation in this study, their representatives for giving permission, and the staff 
from the day-care centers for performing the assessments.

\section{References}

Ahmed, N. and Thornalley, P. J. (2007). Advanced glycation endproducts: what is their relevance to diabetic complications? Diabetes, Obesity and Metabolism, 9, 233-245.

American Psychiatric Association (1994). Diagnostic and Statistical Manual of Mental Disorders (DSM-IV). Washington, DC: American Psychiatric Association.

Andersen, C. K., Wittrup-Jensen, K. U., Lolk, A., Andersen, K. and Kragh-Sørensen, P. (2004). Ability to perform activities of daily living is the main factor affecting quality of life in patients with dementia. Health and Quality of Life Outcomes, 2, 52.

Avery, N. C. and Bailey, A. J. (2005). Enzymic and non-enzymic cross-linking mechanisms in relation to turnover of collagen: relevance to aging and exercise. Scandinavian fournal of Medicine $\mathcal{E}$ Science in Sports, 15, 231-240.

Bird, T. D. (1993). Alzheimer Disease Overview. In R. A. Pagon et al. (eds.). Seattle, WA: GeneReviews [Internet].

Buchman, A. S. and Bennett, D. A. (2011). Loss of motor function in preclinical Alzheimer's disease. Expert Review of Neurotherapeutics, 11, 665-676.

Collin, C., Wade, D. T., Davies, S. and Horne, V. (1988). The Barthel ADL Index: a reliability study. International Disability Studies, 10, 61-63.

Drenth, H., Zuidema, S., Krijnen, W., Bautmans, I., van der Schans, C. and Hobbelen, H. (2017). Advanced glycation end-products are associated with the presence and severity of paratonia in early stage Alzheimer's disease. Fournal of the American Medical Directors Association, in press.

Drenth, H., Zuidema, S., Bunt, S., Bautmans, I., van der Schans, C. and Hobbelen, H. (2016). The contribution of advanced glycation end product (AGE) accumulation to the decline in motor function. European Review of Aging and Physical Activity: Official fournal of the European Group for Research into Elderly and Physical Activity, 13, 3.

Farmer, S. E. and James, M. (2001). Contractures in orthopaedic and neurological conditions: a review of causes and treatment. Disability and Rehabilitation, 23, 549-558.

Folstein, M. F., Folstein, S. E. and McHugh, P. R. (1975). "Mini-mental state". A practical method for grading the cognitive state of patients for the clinician. Fournal of Psychiatric Research, 12, 189-198.

Giebel, C. M., Sutcliffe, C. and Challis, D. (2017). Hierarchical decline of the initiative and performance of complex activities of daily living in dementia. Fournal of Geriatric Psychiatry and Neurology, 30, 96-103.

Graham, J. E., Ostir, G. V., Fisher, S. R. and Ottenbacher, K. J. (2008). Assessing walking speed in clinical research: a systematic review. Fournal of Evaluation in Clinical Practice, 14, 552-562.

Hobbelen, J. S. M., Koopmans, R.T., Verhey, F. R., Habraken, K. M. and de Bie, R. A. (2008). Diagnosing paratonia in the demented elderly: reliability and validity of the paratonia assessment instrument (PAI). International Psychogeriatrics/IPA, 20, 840-852.

Kempen, G. I. J. M. and Doeglas, D. M. and Suurmeijer, Th. P. B. M. (1993). Het Meten Van Problemen met Zelfredzaamheid op Verzorgend en Huishoudelijk Gebied met de GARS: Een Handleiding. 1st edn., Groningen: Noordelijk Centrum voor Gezondheidsvraagstukken, Rijksuniversiteit Groningen.

Li, J., Liu, D., Sun, L., Lu, Y. and Zhang, Z. (2012). Advanced glycation end products and neurodegenerative diseases: mechanisms and perspective. Fournal of the Neurological Sciences, 317, 1-5.

Liu, G. and Liang, K. Y. (1997). Sample size calculations for studies with correlated observations. Biometrics, 53, 937-947.

Magelhaes, P.M., Appell, H. J. and Duarte, J. A. (2008). Involvement of advanced glycation end products in the pathogenesis of diabetic complications: the protective role of regular physical activity. European Review of Aging and Physical Activity, 5, 17-29.

Martyr, A. and Clare, L. (2012). Executive function and activities of daily living in Alzheimer's disease: a correlational meta-analysis. Dementia and Geriatric Cognitive Disorders, 33, 189-203.

Meerwaldt, R. et al. (2004). Simple non-invasive assessment of advanced glycation endproduct accumulation. Diabetologia, 47, 1324-1330.

Metzelthin, S. F. et al. (2010). The psychometric properties of three self-report screening instruments for identifying frail older people in the community. BMC Public Health, $10,176$.

Nenna, A., Spadaccio, C., Lusini, M., Ulianich, L., Chello, M. and Nappi, F. (2015). Basic and clinical research against advanced glycation end products (AGEs): new compounds to tackle cardiovascular disease and diabetic complications. Recent Advances in Cardiovascular Drug Discovery, 10, 10-33.

Payne, G. W. (2006). Effect of inflammation on the aging microcirculation: impact on skeletal muscle blood flow control. Microcirculation, 13, 343-352.

Puyvelde, K., Van, Mets, T., Njemini, R., Beyer, I. and Bautmans, I. (2014). Effect of advanced glycation end product intake on inflammation and aging: a systematic review. Nutrition Reviews, 72, 638-650.

Rahmadi, A., Steiner, N. and Munch, G. (2011). Advanced glycation endproducts as gerontotoxins and biomarkers for carbonyl-based degenerative processes in Alzheimer's disease. Clinical Chemistry and Laboratory Medicine: CCLM/FESCC, 49, 385-391.

Ramakers, I. H. G. B. et al. (2007). Symptoms of preclinical dementia in general practice up to five years before dementia diagnosis. Dementia and Geriatric Cognitive Disorders, 24, 300-306.

Reisberg, B., Ferris, S. H., de Leon, M. J. and Crook, T. (1982). The global deterioration scale for assessment of primary degenerative dementia. The American fournal of Psychiatry, 139, 1136-1139.

Ries, J. D., Echternach, J. L., Nof, L. and Gagnon Blodgett, M. (2009). Test-retest reliability and minimal detectable change scores for the timed "up \& go" test, the six-minute walk test, and gait speed in people with Alzheimer disease. Physical Therapy, 89, 569-579. 
Scherder, E., Eggermont, L., Visscher, C., Scheltens, P. and Swaab, D. (2011). Understanding higher level gait disturbances in mild dementia in order to improve rehabilitation: "last in-first out." Neuroscience and Biobehavioral Reviews, 35, 699-714.

Sternberg, S. A., Wershof Schwartz, A., Karunananthan, S., Bergman, H. and Mark Clarfield, A. (2011). The identification of frailty: a systematic literature review. Fournal of the American Geriatrics Society, 59, 2129-2138.
Whitson, H. E. et al. (2014). Serum carboxymethyl-lysine, disability, and frailty in older persons: the Cardiovascular Health Study. The fournals of Gerontology. Series A, Biological Sciences and Medical Sciences, 69, 710-716.

World Health Organisation (2014). The Anatomical Therapeutic Chemical Classification System with Defined Daily Doses (ATC/DDD). Available at: http://www.who.int/classifications/atcddd/en/ [Accessed September 1, 2014]. 